


\title{
Efecto del reforzamiento sismo-resistente sobre el comportamiento dinámico de una edificación afectada por el sismo de Pizarro
}

\author{
Helene Tischer * ${ }^{\S}$, Johannio Marulanda *, Peter Thomson * \\ * Escuela de Ingeniería Civil y Geomática, Facultad de Ingeniería, \\ Universidad del Valle, Cali, Colombia \\ §e-mail: helenet@yahoo.com
}

(Recibido: Abril 29 de 2006 - Aceptado: Septiembre 27 de 2006)

\section{Resumen}

El 15 de noviembre de 2004, la ciudad de Cali se vio afectada por el sismo de Pizarro. Varias edificaciones sufrieron daños y alrededor de 20 de estas sufrieron daños considerables, lo que hizo necesaria su intervención. Algunas de las edificaciones afectadas habían sido reforzadas con anterioridad al sismo, lo cual hace dudar acerca de la efectividad del reforzamiento sismo-resistente en los parámetros dinámicos de una edificación. En este artículo se describen los ensayos de vibraciones ambientales llevadas a cabo en una edificación en Cali, con posterioridad al sismo y tanto antes como después de su reforzamiento sismo-resistente. Se presentan los resultados del procesamiento digital de los registros de aceleración, con los cuales es posible analizar los cambios en los parámetros dinámicos producidos por el reforzamiento. Específicamente, se observó que las frecuencias naturales de la edificación aumentaron entre 30 y $50 \%$ con el reforzamiento.

Palabras clave: Reforzamiento sísmico, Estudio dinámico, Frecuencias naturales, Vibraciones ambientales.

\section{Effect of seismic retrofit on the dynamic behavior of a building damaged by the Pizarro earthquake}

\begin{abstract}
On November 15, 2004, the city of Cali was affected by the Pizarro earthquake. Several buildings were damaged and about 20 of these suffered considerable damage. Before the earthquake, some of the buildings had been seismically retrofitted, which raises questions regarding the effectiveness of the retrofit with respect to the dynamic parameters of a building. This paper describes a series of ambient vibration tests that were conducted on a building in Cali after the Pizarro earthquake both prior to and after seismic retrofit. Changes in the dynamic parameters of the building due to the retrofit were analyzed using digital processing of the acceleration measurements. Specifically, an increase of the natural frequencies of the buildings in the range of $30 \%$ to $50 \%$ was observed after seismic retrofit.
\end{abstract}

Keywords: Seismic retrofit, Dynamic studies, Natural frequencies, Ambient vibrations. 


\section{Introducción}

El 15 de noviembre de 2004, la ciudad de Cali se vio afectada por el sismo de Pizarro, con epicentro frente a las costas del sur de Chocó y magnitud de 7.2 MW [http://osso.univalle.edu.co (2005)]. En esta ocasión, se observó que varias edificaciones sufrieron daños, pudiéndose destacar alrededor de 20 edificios que sufrieron daños considerables. Algunas de las edificaciones afectadas habían sido intervenidas con anterioridad, lo cual hace dudar, a la vista de las normativas existentes, acerca de la efectividad de las intervenciones realizadas.

El Estado colombiano aprobó, en 1997, la Ley No. 400 por la cual se adoptan las Normas Colombianas de Diseño y Construcción SismoResistente (NSR-98), que "establecen criterios y requisitos mínimos para el diseño, construcción y supervisión técnica de edificaciones" [Asociación Colombiana de Ingeniería Sísmica (2003)]. Las edificaciones afectadas por el último sismo, que han sido o están siendo intervenidas, deben cumplir con esta normativa.

El diseño del reforzamiento se basa generalmente en modelos analíticos tridimensionales de las estructuras a intervenir. Estos modelos son construidos mediante programas de computador comerciales verificados, usualmente basados en el método de los elementos finitos. Sin embargo, en muy pocas ocasiones, los modelos analíticos son validados para garantizar que reproducen de manera confiable el comportamiento de la estructura real.

La alternativa más confiable para la validación de un modelo analítico es la realización de ensayos experimentales que confirmen consideraciones del modelo analítico que posiblemente no reproducen las condiciones reales en la estructura, como, por ejemplo, la resistencia de los materiales, las conexiones entre elementos y el aporte en rigidez de elementos no estructurales. Los ensayos dinámicos experimentales permiten caracterizar el comportamiento dinámico de una estructura y compararlo con el comportamiento dinámico del modelo analítico para realizar ajustes y garantizar su validez. Finalmente, mediante un modelo analítico confiable es posible garantizar que la estructura tendrá un buen desempeño frente a las solicitaciones a las que se verá sometida durante su vida útil.

\section{Metodología}

\subsection{Identificación de la edificación}

Para el presente estudio, se seleccionó una de las edificaciones afectadas por el sismo de Pizarro: la torre A del Conjunto Residencial Torres de la Plazuela, localizada en la carrera 40 \# 6-24/50. Las condiciones del suelo en el sitio, correspondientes al abanico del río Cañaveralejo, generan amplificación de las ondas sísmicas, especialmente para periodos largos, superiores a 1 seg (Instituto Colombiano de Geología y Minería, 2004). Esto explica la concentración de edificaciones similares afectadas en esta área por el sismo de Pizarro.

Se trata de una edificación de 16 pisos de altura y dos sótanos de parqueaderos, cuyo uso es residencial. La altura total de la edificación es de aproximadamente $50 \mathrm{~m}$ desde el nivel del suelo y su área en planta aproximadamente $275 \mathrm{~m}^{2}$. El sistema estructural original consistía en pórticos y pantallas de concreto reforzado. Las losas del edificio son aligeradas y armadas en una dirección, con vigas de carga de 40 por $50 \mathrm{~cm}$, y vigas de amarre de 30 por $50 \mathrm{~cm}$.

Después del sismo, y a causa de los daños sufridos por la edificación, la misma se reforzó usando pantallas de concreto reforzado, arriostramiento en acero y colocación de vigas en zonas críticas, tanto metálicas como de concreto reforzado. Las pantallas empleadas son de $25 \mathrm{~cm}$ de espesor con columnas de confinamiento a ambos lados, aledañas a las pantallas existentes y ancladas a las mismas. Lo anterior se realizó para que las pantallas existentes y las nuevas trabajaran como un solo elemento, restándoles esbeltez, ya que se pasó de una sección de $25 \mathrm{~cm}$ de espesor a una de $50 \mathrm{~cm}$. Además, se reemplazaron los muros agrietados por el sismo, de mampostería tradicional de ladrillo de arcilla por cerramientos livianos del tipo Drywall. Se conservaron algunos muros, localizados en los últimos pisos, debido a que no sufrieron daños. 


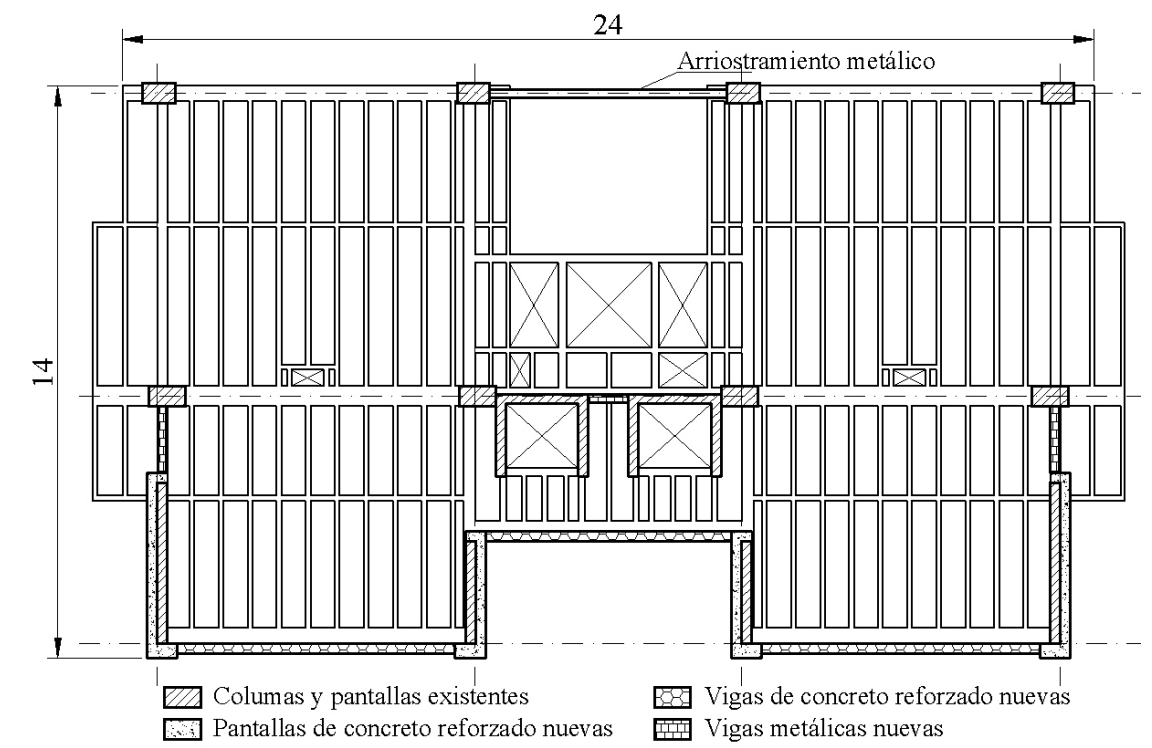

Figura 1. Planta tipo esquemática del edificio, basada en planos del ingeniero Diego Renjifo.
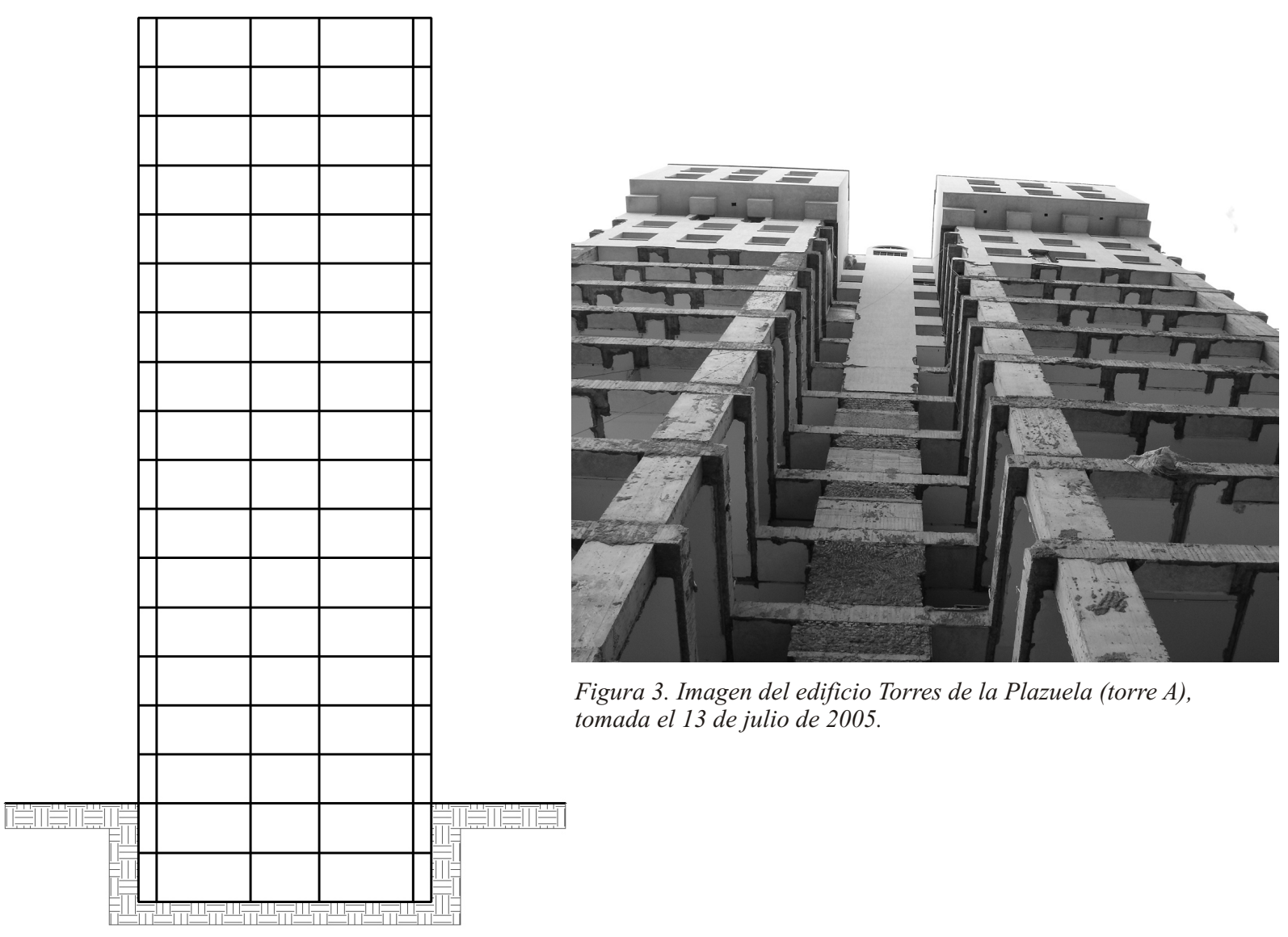

Figura 3. Imagen del edificio Torres de la Plazuela (torre A), tomada el 13 de julio de 2005.

Figura 2. Alzada esquemática del edificio. 
En la Figura 1 se presenta la planta del piso tipo de la edificación, donde se detalla tanto el sistema estructural existente como el refuerzo propuesto, $y$ en la Figura 2 se detalla el esquema en altura de la edificación. En la Figura 3 se presenta una imagen de la edificación después del sismo de Pizarro, tomada el 13 de julio de 2005. En ese momento los muros afectados por el sismo ya habían sido retirados, pero no se había comenzado con el reforzamiento estructural.

\subsection{Definición de los indicadores}

Para constatar el efecto del reforzamiento sobre el comportamiento dinámico de la edificación, se tomaron como indicadores las principales frecuencias naturales de la edificación. Estos parámetros están relacionados directamente con la rigidez y la masa de la estructura. Un aumento de las frecuencias naturales con el reforzamiento, considerando que la masa no varía, sería entonces indicativo de una mayor rigidez de la estructura ante futuros eventos sísmicos, que es el efecto que tradicionalmente se busca en los procesos de reforzamiento. Esto lo convierte en un indicador apropiado para medir el efecto del reforzamiento planteado. En este caso, los registros previos al reforzamiento se realizaron cuando ya se habían retirado de la edificación los muros agrietados; y los registros posteriores al reforzamiento se realizaron con la presencia de las nuevas pantallas, el arriostramiento metálico y parte de las divisiones livianas. Por lo tanto, la masa del sistema aumentó entre las mediciones dinámicas, lo que produce una disminución en las frecuencias naturales. Si la rigidez aumentara, compensaría este efecto.

Debe aclararse que la rigidez, cuyo aumento es asociado tradicionalmente con un mejor comportamiento ante eventos sísmicos, no debe ser el único parámetro a evaluar en un estudio de reforzamiento sismo-resistente; la resistencia y la ductilidad son de vital importancia y van de la mano con la rigidez. En un análisis modal cronológico o espectral es muy importante considerar los efectos locales del suelo, pues las características dinámicas del sitio se reflejan directamente en la magnitud de la carga sísmica que afectará la edificación, lo que lleva a considerar su capacidad de disipación de energía (ductilidad) y la capacidad de sus elementos estructurales (resistencia).

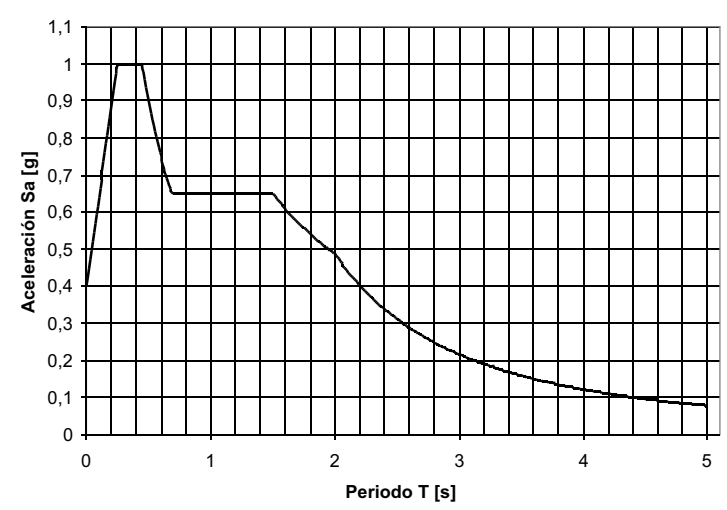

Figura 4. Espectro de diseño de la zona $4 C$, de Cañaveralejo, de acuerdo a información de la microzonificación sísmica de Cali.

En este caso, para tener en cuenta los efectos locales, se consideró el espectro de diseño propuesto para la zona 4C: abanico de Cañaveralejo (Calisísmica, 2006), que se presenta en la Figura 4.

\subsection{Identificación modal}

Se realizaron mediciones de vibraciones ambientales de la estructura mediante el sistema portátil del Laboratorio de Ingeniería Sísmica y Dinámica Estructural (LINSE) de la Universidad del Valle (ver Figura 5).

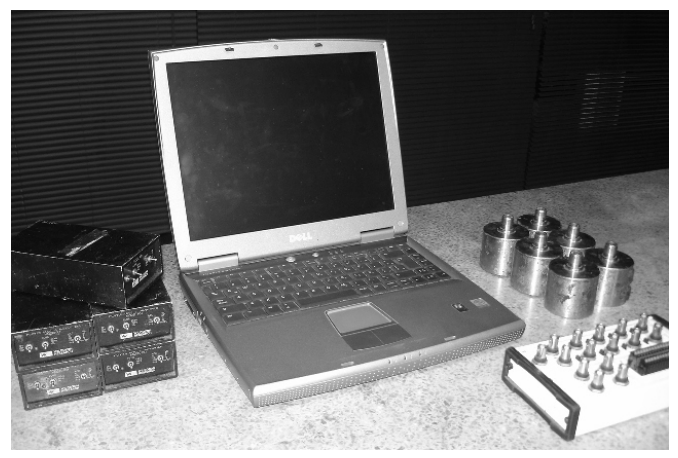

Figura 5. Sistema portátil del Laboratorio de Ingeniería Sísmica y Dinámica Estructural (LINSE) de la Universidad del Valle. 
El sistema portátil del LINSE consta de 8 acelerómetros sísmicos Wilcoxon Research Model 731A, cada uno con un amplificador Wilcoxon Research Model P31. Los acelerómetros tienen una sensibilidad de $10 \mathrm{~V} / \mathrm{g}$ y junto con los amplificadores se puede obtener una sensibilidad total de $10 \mathrm{~V} / \mathrm{g}, 100 \mathrm{~V} / \mathrm{g}$ ó $1000 \mathrm{~V} / \mathrm{g}$. Las unidades WR Model P31 amplifican las señales análogas y proveen un filtrado integral con un ancho de banda nominal (menos $3 \mathrm{~dB}$ ) entre 0.05 y $450 \mathrm{~Hz}$. Las señales de los acelerómetros se transmiten mediante cables coaxiales a una caja de conexiones National Instruments NI BNC 2110 y luego a un computador portátil, donde se lleva a cabo la conversión de la señal analógica a digital con una tarjeta de adquisición de datos NI DAQCard 6024E con 12 bits de resolución. La adquisición de datos se efectuó con la caja de herramientas (Toolbox) para adquisición de datos del paquete matemático MATLAB (The MathWorks, Inc., 2003a).

Las mediciones de vibraciones ambientales se realizaron ubicando los sensores en puntos clave de la estructura, seleccionados teniendo en cuenta las formas modales esperadas, y en función de la información que se desea obtener (i.e., el balanceo).

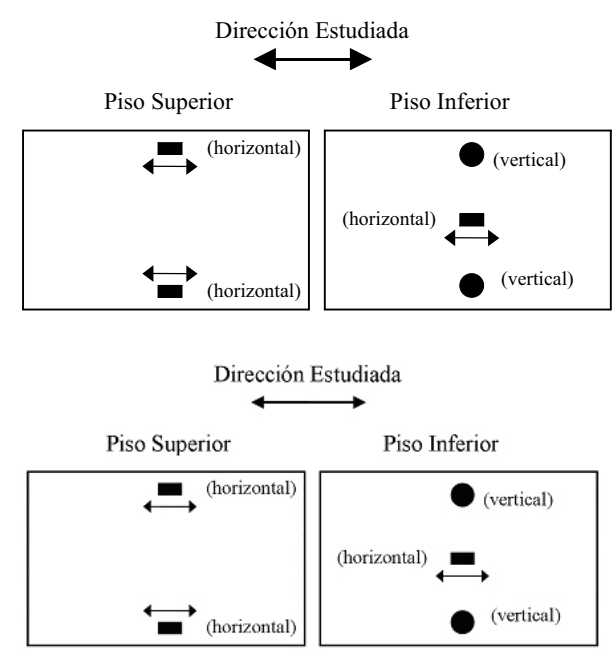

Figura 6. Ubicación esquemática de los acelerómetros sísmicos para los registros de vibraciones ambientales.

En cada caso se tomaron dos registros de 10 minutos de duración, con una tasa de muestreo de
256 muestras por segundo, en las dos direcciones principales de la edificación. En cada registro se colocaron dos acelerómetros horizontales en el último nivel y tres en el primero (dos verticales y uno horizontal), tal como se indica esquemáticamente en la Figura 6. Esta configuración se usó independientemente para las dos direcciones principales de la edificación.

Los registros previos al reforzamiento se realizaron el 13 de julio de 2005, 7 meses después de ocurrido el sismo de Pizarro (ver Figura 3). Para esta fecha, ya se habían demolido los muros agrietados, los cuales fueron la totalidad de los muros de $\operatorname{los}$ pisos 1 a 10 y aproximadamente $80 \%$ de los muros de los pisos 11 a 13. En los pisos $15 \mathrm{y}$ 16 los muros no se vieron afectados por el sismo, pero se habían demolido en las zonas donde se colocarían pantallas de concreto reforzado. Los registros posteriores al reforzamiento estructural se realizaron el 22 de febrero de 2006. Para esa fecha, ya se había concluido el reforzamiento estructural de la edificación, incluyendo el arriostramiento metálico instalado en la fachada posterior de la edificación, que se observa en la Figura 7, y se había instalado parte de las divisiones livianas.

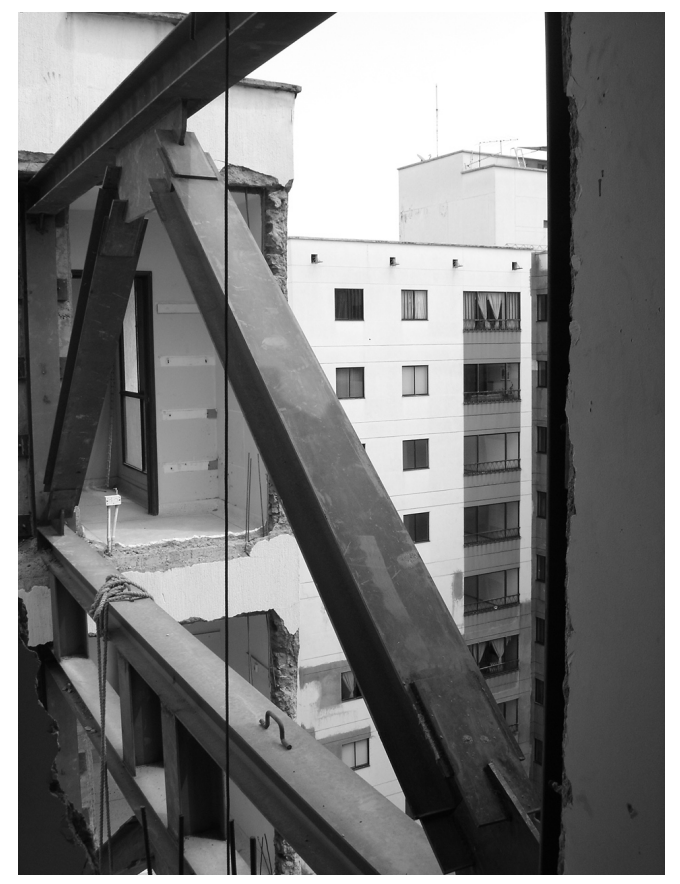

Figura 7. Arriostramiento metálico instalado en la fachada posterior de la edificación. 


\subsection{Procesamiento de las señales}

Los registros se analizaron mediante la caja de herramientas (Toolbox) para procesamiento digital de señales del paquete matemático MATLAB (The MathWorks, Inc., 2003a), identificándose dos fases: el preproceso y el análisis. El procedimiento seguido se presenta de manera esquemática en la Figura 8.

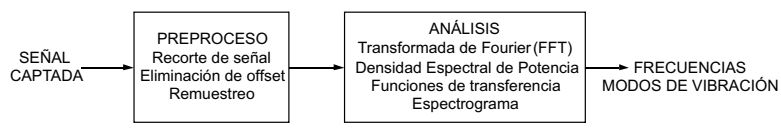

Figura 8. Esquema del procesamiento digital aplicado a las señales captadas.

En el preproceso se acondicionó la señal captada para su valoración, recortándola para excluir zonas donde algún canal se saturara. También se eliminó el desfase (offset) constante o variable, que presentaban algunos canales. Por último, se remuestreó la señal a una frecuencia de $10 \mathrm{~Hz}$, teniendo en cuenta que las frecuencias de interés esperadas para el edificio se encuentran entre 0 y $5 \mathrm{~Hz}$.

En la etapa de análisis se calcularon la densidad espectral de potencia, las funciones de transferencia entre canales y los espectrogramas. La densidad espectral de potencia para una señal discreta en el tiempo y periódica se deduce a partir de la potencia media de la misma, definida según Proakis \& Manolakis (1998) por medio de la Eq. (1):

$$
P_{x}=\frac{1}{N} \sum_{n=0}^{N-1}|x(n)|^{2}=\sum_{k=0}^{N-1}\left|c_{k}\right|^{2}
$$

donde $N$ es el periodo de la señal y $x(n)$ es la señal periódica discreta en el tiempo, representada a través de series de Fourier como

$$
x(n)=\sum_{k=0}^{N-1} c_{k} e^{j 2 \pi k n / N}
$$

donde $c_{k}$ son los coeficientes de la representación en serie.
La secuencia $\left|c_{k}\right|^{2}$ se denomina densidad espectral de potencia, y representa la distribución de la potencia en función de frecuencia. La densidad espectral de potencia se estimó usando el método de Welch (The MathWorks, Inc., 2003b), con 1024 datos para el cálculo de la transformada rápida de Fourier y un traslapo de 50\% usando la ventana tipo Hanning. Los picos en el gráfico de densidad espectral de potencia permiten la identificación de las frecuencias de los modos fundamentales.

Las funciones de transferencia representan la relación entre una señal de entrada y una de salida, de manera lineal e invariante en el tiempo, y se estimaron usando el método del periodograma promedio de Welch (The MathWorks, Inc., 2003b). Se usaron para asociar los picos de frecuencia identificados con los modos de vibración respectivos, estableciendo si los canales analizados se encuentran en fase o desfase para las frecuencias de interés, y estimando la relación de amplitudes.

Un espectrograma es una gráfica de tres dimensiones que muestra la variación en tiempo y frecuencia del contenido de energía de una señal, mediante la variación cromática de la amplitud. Permite identificar frecuencias predominantes que no son constantes en el tiempo y que por tanto no son de interés para el análisis. Estas contribuciones en frecuencia pueden proceder de actividades de obra realizadas durante la toma de los registros, como la demolición de muros y el vertimiento de escombros desde pisos superiores. Para el cálculo de los espectrogramas se usó la transformada rápida de Fourier, calculada para 1024 datos y un traslapo de $50 \%$ con una ventana tipo Hanning (The MathWorks, Inc., 2006).

\section{Resultados y discusión}

Las frecuencias naturales se identificaron localizando los picos más destacados en los gráficos de densidad espectral de potencia de las dos direcciones principales de la edificación, tanto antes como después del reforzamiento, para todos los registros. Se observaron dos picos de interés en todos los casos, tal como se observa en las Figuras 9 a 12. 


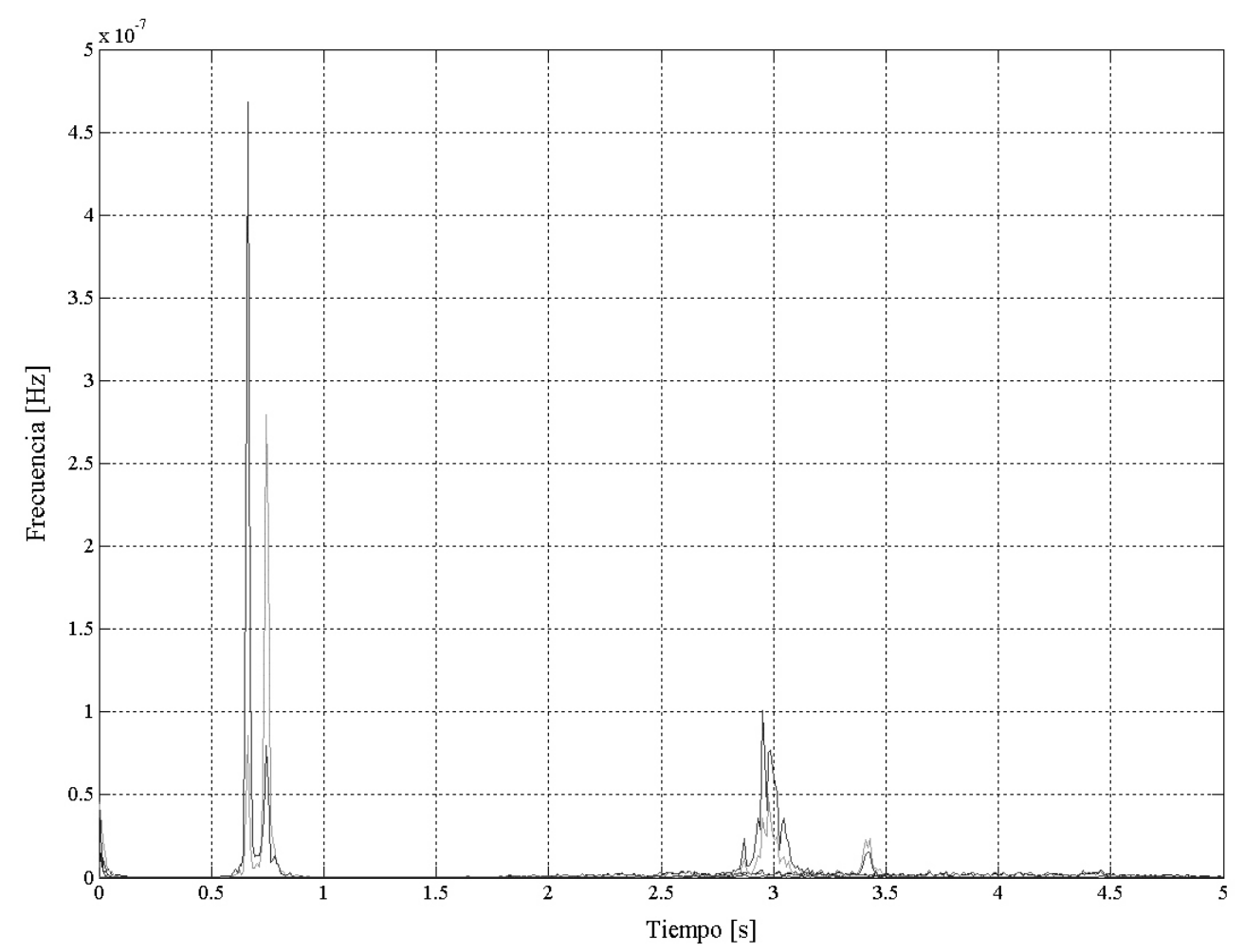

Figura 9. Densidad de potencia espectral, dirección norte-sur, antes del reforzamiento.

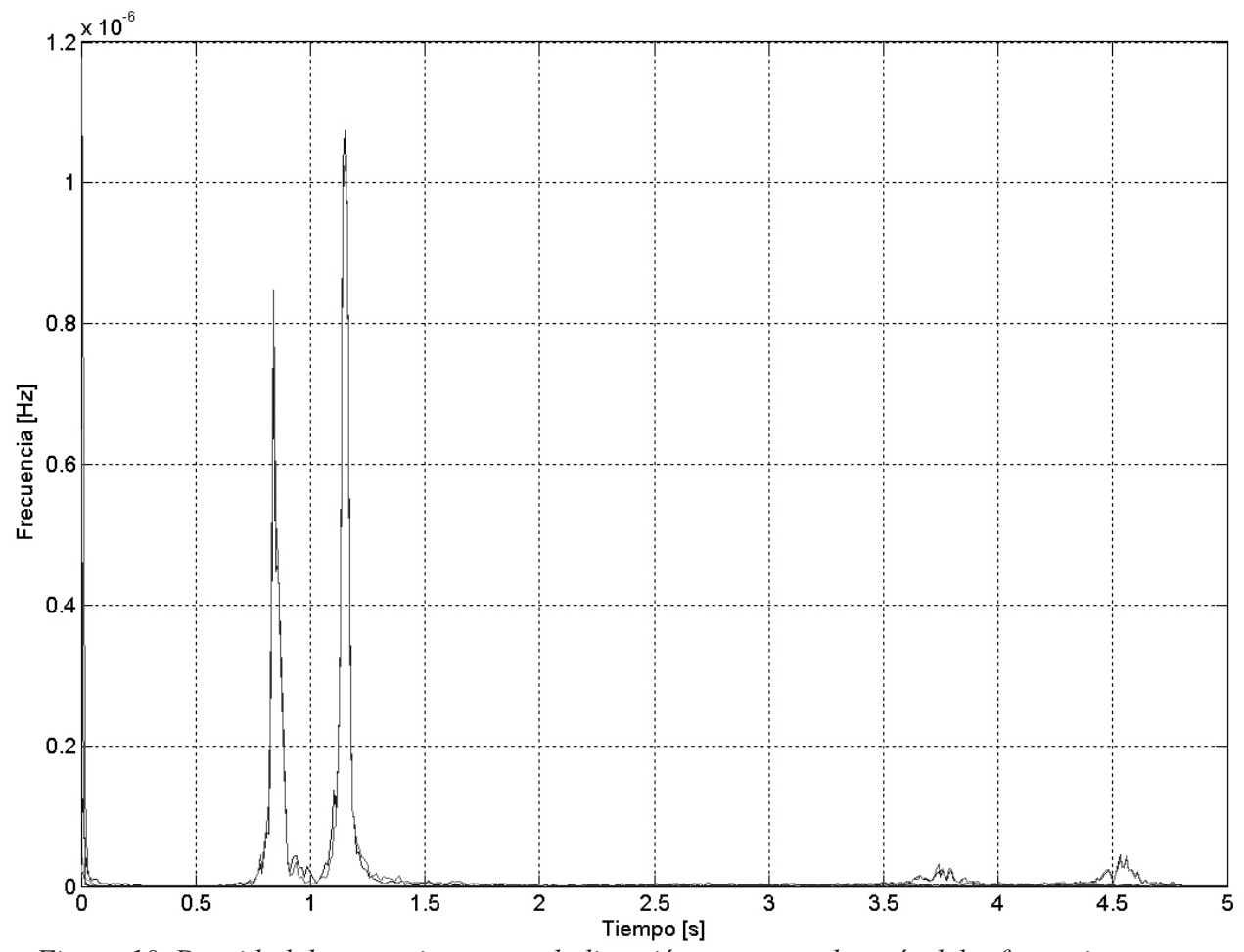

Figura 10. Densidad de potencia espectral, dirección norte-sur, después del reforzamiento. 


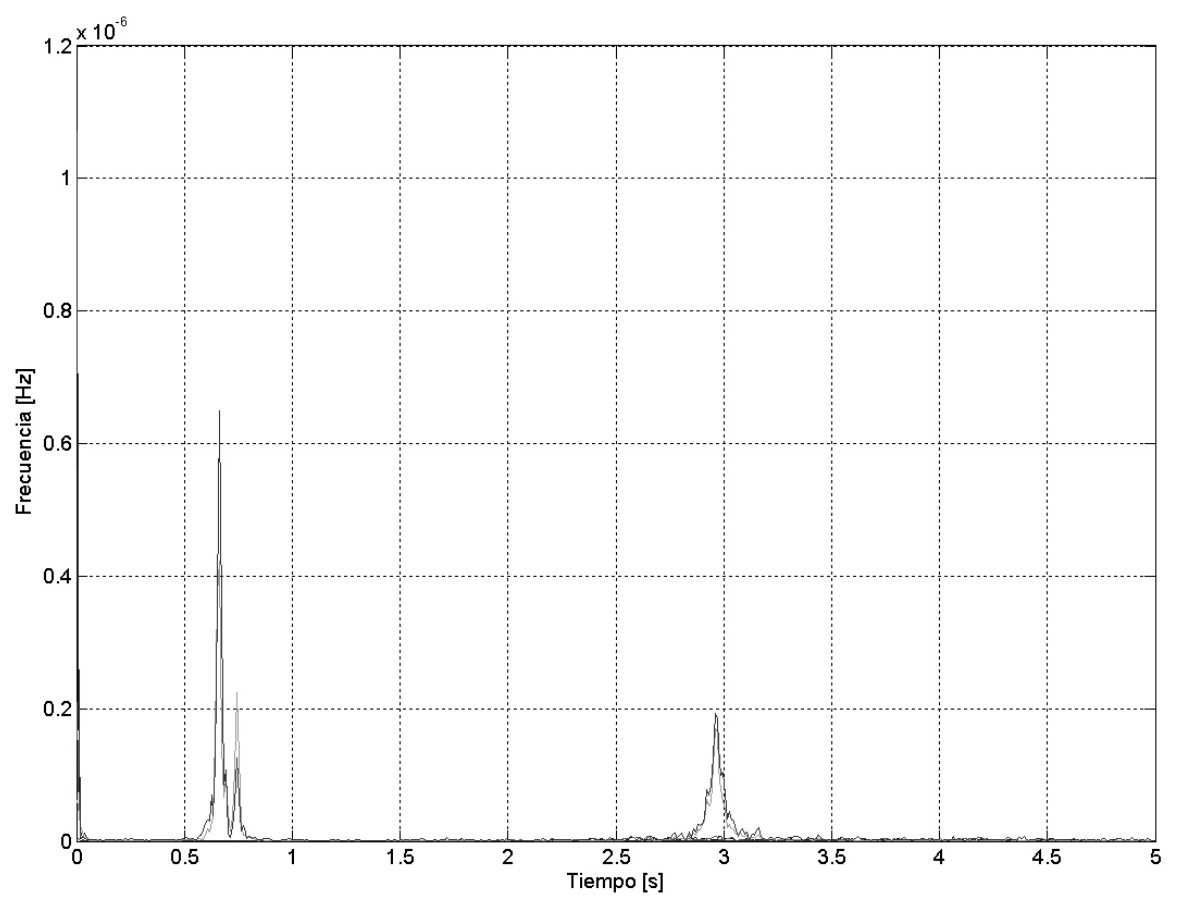

Figura 11. Densidad de potencia espectral, dirección este-oeste, antes del reforzamiento.

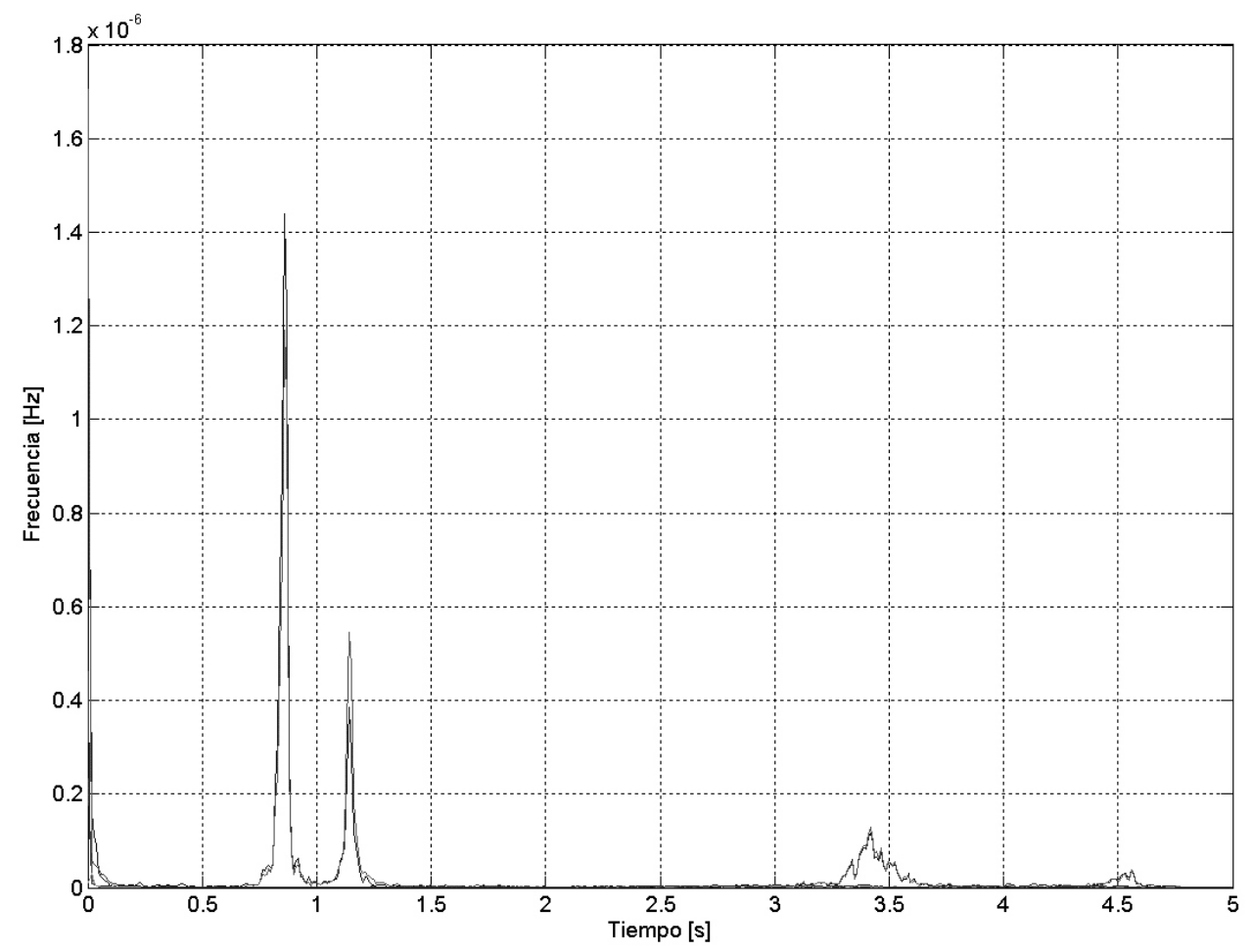

Figura 12. Densidad de potencia espectral, dirección este-oeste, después del reforzamiento. . 
En ellas se representa la densidad espectral de potencia de un registro previo al reforzamiento y uno posterior al mismo, para las dos direcciones principales de la edificación. Los canales que presentan picos pronunciados son los que corresponden a sensores localizados en el último piso, mientras que los otros corresponden a los registros en el nivel del suelo y, por tanto, representan principalmente el contenido frecuencial del movimiento del terreno. Los dos canales superiores tienen un comportamiento tan similar que sus gráficas se superponen en la mayor parte de la figura.

Con la ayuda de las Figuras 9 y 11, correspondientes a la densidad de potencia espectral de todos los canales antes del reforzamiento, y las funciones de transferencia entre los mismos canales, se identificaron los dos primeros modos de flexión en ambas direcciones con frecuencias naturales asociadas cercanas a $0.6 \mathrm{~Hz}$, y el primer modo torsional con frecuencia $0.7 \mathrm{~Hz}$, aproximadamente. Para la identificación de los modos se analizaron en total 20 funciones de transferencia por registro, tanto entre cada uno de los canales del primer piso y los del último, como entre los dos del último piso, para un total de 80 funciones estudiadas. En la figura 13 se presenta como ejemplo la función de transferencia para los dos canales del último piso, para uno de los registros en la dirección norte-sur antes del reforzamiento. En general, en cada figura el primer pico corresponde a las dos frecuencias de los modos de flexión muy cercanas entre sí y el segundo al mismo modo torsional.
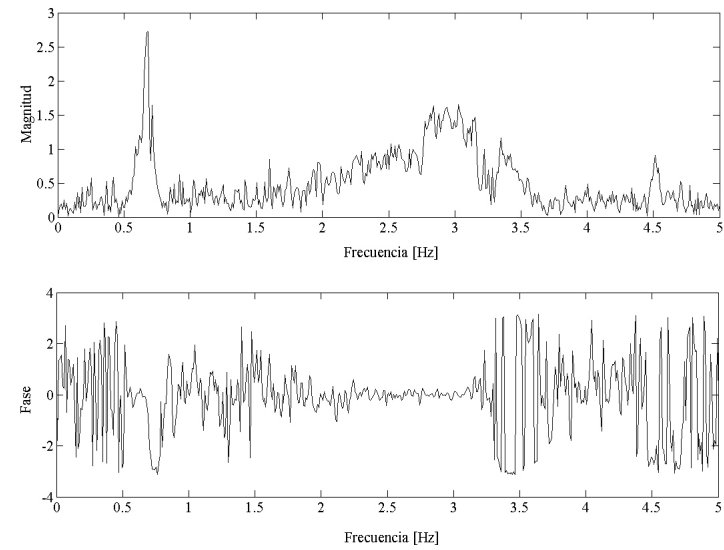

Figura 13. Función de transferencia entre los dos canales ubicados en el piso 16, dirección norte-sur, previo al reforzamiento.

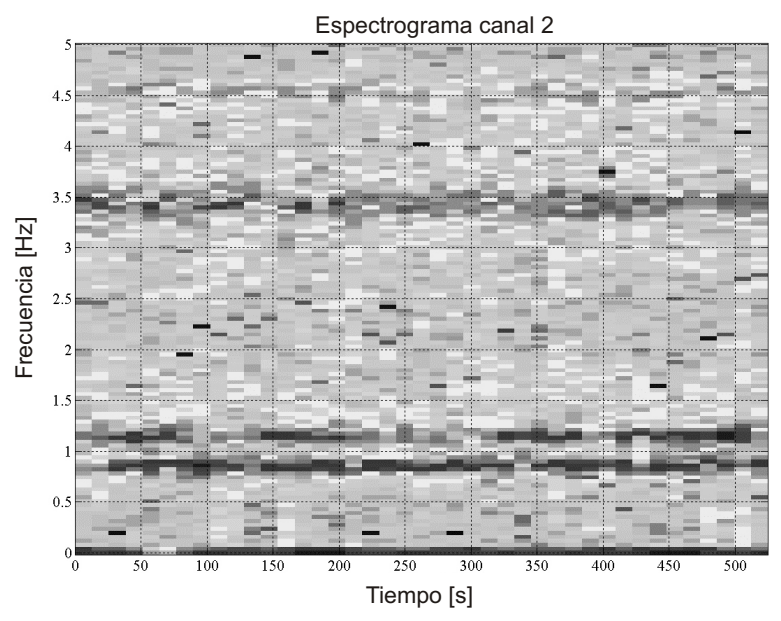

Figura 14. Espectrograma de uno de los canales del último piso, dirección norte-sur, después del reforzamiento.

Con ayuda de las figuras 10 y 12 , correspondientes a la densidad de potencia espectral de todos los canales después del reforzamiento, y las funciones de transferencia entre los mismos canales, se identificaron los dos primeros modos de flexión en ambas direcciones con frecuencias naturales asociadas cercanas a $0.8 \mathrm{~Hz}$, y el primer modo torsional con frecuencia $1.2 \mathrm{~Hz}$, aproximadamente.

En todos los casos se corroboró que los contenidos frecuenciales fueran estables en el tiempo. Para esto se generó el espectrograma para cada canal, en cada uno de los registros, para un total de 20 espectrogramas analizados. Como ejemplo, en la Figura 14, se presenta el espectrograma de uno de los canales del último piso, en uno de los registros norte-sur tomado después del reforzamiento.

Los resultados de la identificación modal son consecuentes con lo establecido en las Normas Colombianas de Diseño y Construcción Sismo Resistente (NSR-98) [Asociación de Ingeniería Sísmica (2003)], que en su Capítulo A.4 establece que el periodo fundamental aproximado de una edificación de pórticos de concreto reforzado resistentes a momentos, se puede calcular según la Eq.(3):

$$
T_{a}=0.08 \cdot h_{n}^{3 / 4}
$$


donde $T_{a}$ es el periodo fundamental aproximado (en s) y $h_{n}$ es la altura (en m), medida desde la base hasta el último piso de la edificación.

Con lo anterior, se puede calcular la frecuencia fundamental aproximada de la edificación como $0.66 \mathrm{~Hz}$, valor muy cercano a lo obtenido experimentalmente.

Es de destacar que las frecuencias obtenidas en ambos sentidos de la edificación son muy similares, lo que se atribuye a la forma regular de la estructura. Aunque se observa una ligera diferencia, esta no es significativa para el error manejado.

Los valores de los periodos fundamentales obtenidos experimentalmente, antes y después del reforzamiento, se usaron para entrar al espectro de diseño (ver Figura 4) establecido por la microzonificación sísmica de Cali para la zona 4C: abanico de Cañaveralejo (Calisísmica, 2006). Con un periodo previo al reforzamiento de $1.70 \mathrm{~s}$, se tiene una aceleración de $0.57 \mathrm{~g}$ (donde $g$ es la aceleración de la gravedad). Después del reforzamiento el periodo bajó a $1.25 \mathrm{~s}$, obteniendo del espectro una aceleración de $0.65 \mathrm{~g}$; lo que corresponde a un aumento en la aceleración sísmica de aproximadamente $14 \%$.

Además de los picos pronunciados analizados anteriormente, localizados en frecuencias coherentes con las características de la edificación, se observaron de manera recurrente picos en aproximadamente $3 \mathrm{~Hz}$ antes del reforzamiento y de $3.5 \mathrm{~Hz}$ posterior al mismo, junto con otros de frecuencias mayores, que pueden corresponder a modos de vibración superiores o a ruido en la señal.

\section{Conclusiones}

Con las mediciones dinámicas realizadas los días 13 de julio de 2005 y 22 de febrero de 2006 en el edificio Torres de la Plazuela (torre A) se identificaron los primeros tres modos de vibración y sus frecuencias naturales asociadas, antes y después del reforzamiento. Los dos primeros tienen una frecuencia aproximada de $0.6 \mathrm{~Hz}$ antes del reforzamiento y $0.8 \mathrm{~Hz}$ después del mismo. Son modos de flexión, en las dos direcciones principales de la edificación. El otro modo tiene una frecuencia de aproximadamente $0.7 \mathrm{~Hz}$ antes del reforzamiento y de $1.2 \mathrm{~Hz}$ después, y corresponde a un modo torsional.

Se puede concluir que las frecuencias naturales aumentaron entre 30 y $50 \%$ con el reforzamiento. Este aumento es indicativo de la rigidización de la edificación, tal como se esperaba tras la intervención y teniendo en cuenta que el cambio en la masa es muy pequeño. El aumento de masa producirá una disminución de las frecuencias, o sea que produce el efecto contrario a la rigidización.

Un efecto propio de la rigidización de la edificación, y consiguiente aumento en las frecuencias naturales, es que la aceleración sísmica aumenta. En el caso particular de la edificación estudiada, y tomando como espectro de diseño el recomendado de acuerdo con la microzonificación sísmica de Cali (Calisísmica, 2006), este aumento fue de aproximadamente $14 \%$. Esto es evidentemente desfavorable para la estructura, aunque el comportamiento final dependerá también de otros factores.

Aparte de la rigidización ya mencionada, con la intervención se logró darle mayor simetría a la estructura, reduciendo problemas asociados a la torsión. Se redujo la esbeltez de las pantallas, pasando de una sección de $25 \mathrm{~cm}$ de espesor a una de $50 \mathrm{~cm}$, y se las conectó con vigas más resistentes, lo que mejora su funcionamiento tanto individualmente como en conjunto con el resto de la estructura. Por último, se redujo el peso total del edificio, reemplazando los muros tradicionales de ladrillo por muros livianos. Considerando que los muros tradicionales pueden pesar alrededor de $270 \mathrm{~kg} / \mathrm{m}^{2}$, comparado con el peso de las divisiones tipo Drywall, que pueden estar alrededor de $30 \mathrm{~kg} / \mathrm{m}^{2}$, esto implica una disminución de casi $90 \%$ del peso de divisiones. Ya que la cortante basal se puede calcular como la aceleración multiplicada por el peso de la edificación (Asociación Colombiana de Ingeniería Sísmica, 2003), la disminución del peso reduce el impacto de un evento sísmico futuro. 
Cabe aclarar que, en los ensayos de vibraciones ambientales, se registran los efectos que las vibraciones de baja amplitud tienen sobre la estructura. Las solicitaciones a la edificación durante la ocurrencia de un evento sísmico son de diferente naturaleza, con amplitudes mucho mayores. Esto, junto a la no linealidad en el sistema suelo-estructura para amplitudes grandes, hacen que las frecuencias puedan variar (Trifunac et al., 2001a; Trifunac et al., 2001b).

\section{Referencias bibliográficas}

Asociación Colombiana de Ingeniería Sísmica (2003). Normas colombianas de diseño y construcción sismo-resistente NSR-98.Bogotá: 3R Editores Ltda.

Calisísmica. (2006). Microzonificación sísmica de Cali. http://www.calisismica.com/propos.htm\#

Instituto Colombiano de Geología y Minería (2004). El sismo de Pizarro (Bajo Baudó, Chocó) del 15 de noviembre de 2004: parámetros de la fuente, movimiento fuerte $y$ daños. Bogotá: Instituto Colombiano de Geología y Minería.
Proakis, J. G.; \& Manolakis, D. G. (1998). Tratamiento digital de señales. Madrid: Prentice Hall.

The MathWorks, Inc. (2003a). MATLAB. Natick: The MathWorks, Inc.

The MathWorks, Inc. (2003b). Signal processing toolbox for use with MATLAB. Natick: The MathWorks, Inc.

Trifunac, M. D., Ivanovic, S. S., \& Todorovska, M. I. (2001a). Apparent periods of a building. I: Fourier analysis. Journal of Structural Engineering 127 (5), 517-526.

Trifunac, M. D., Ivanovic, S. S., \& Todorovska, M. I. (2001b). Apparent periods of a building. II: Time-frequency analysis. Journal of Structural Engineering 127 (5), 527-537. 